\title{
MODELO RECURSIVO DE GESTIÓN HOLÍSTICA DE ACTIVOS Y PASIVOS (GHAP) PARA OPTIMIZAR LOS RESULTADOS ECONÓMICO FINANCIEROS EN LAS CAJAS MUNICIPALES DE AHORRO Y CRÉDITO (CMACs) DEL PERÚ - 2009
}

\author{
Autor: Fidel Carita Monroy' \\ Universidad Privada de Tacna \\ E-mail: fcarita@esan.org.pe
}

\section{RESUMEN}

El presente trabajo de investigación plantea un modelo de dirección, de gestión que permite optimizar los rendimientos del capital invertido, como resultado de un manejo adecuado de los flujos de activos y pasivos financieros involucrados en la esencia del negocio: Captaciones y colocaciones.

En los últimos 10 años las CMACs en el Perú muestran decrecimiento en su rentabilidad; razón que hace necesaria la implementación de un modelo de dirección que permita corregir este problema.

En este contexto se plantea el modelo GHAP, como un proceso holistico, sistemático y continuo en su estructura y en cada una de sus partes. Por lo tanto, obedece a una perspectiva integral (del todo), ordenada e iterativa.

Este modelo es ergódico y autopoiético; se establece y sustenta en tres componentes: de procesos, de redes y estructuras funcionales y de recursividad.

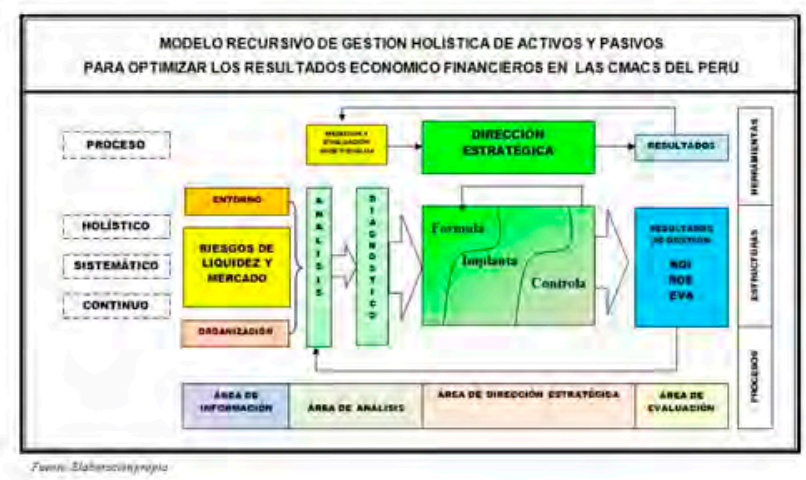

\section{PALABRAS CLAVE:}

Holístico: Es la visión integral, como un todo.

Recursivo: Relacionado a la retroalimentación y del control por auditorías en tiempo real.

Autopoiético: Característica de la clase mundial en la gestión por temporalidades, que consiste en ser disciplinario, multidisciplinario y transdisciplinario.

Iterativo: que sucede una y otra vez.

\section{ABSTRACT}

This research work proposes a model for direction and management that allows optimize the profits of capital invested as a result of an adequate management of active and poassive capital flow in the core of business: receptions and collocations.

In the last 10 years CMACS in Peru have shown a decrease in profitability, that is why it is necessary to implement a model to allow the correction of such problem.

In this connection we propose the GHAP model as a holistic , systemic and continuing process in its structure and each of its parts. It fulfills the model of an integral perspective (a whole) which is ordered and iterative.

This is an ergodic and autopoietic model. It is established and founded on three main components: processes, networks and functional structures as well as recursivity.

\section{KEYWORDS:}

Holístic: It is an integral vision, as a whole.

Recursive: Related to feedback and audited control in real time,

Autopoietic: A characteristic of world class in management by seasonalities, which consists in being disciplinary, multidisciplinary and transdisciplinary as well.

Iterative: That it happens again and again. 


\section{INTRODUCCIÓN}

El sistema financiero nacional es uno de los soportes importantes de la economía del país. Un comportamiento ordenado y eficiente de los involucrados en el sistema permitirá que el escenario económico muestre variables mucho más favorables para su desarrollo.

Dentro de este contexto, las Cajas Municipales de Ahorro y Crédito en el Perú, desde la década de los noventa han tomado importancia con su participación en el mercado financiero, consolidándose en el segmento microfinanciero, en la última década; haciendo microfinanzas.

Siendo sus clientes, principalmente, los micro y pequeños empresarios asi como parte del sector informal.

Sin embargo, ese crecimiento no ha ido acompañado por diferentes líneas de producción ni de decisión, ni de gestión, de un mejoramiento en la misma proporción de crecimiento de cada una de ellas; disminuyendo sus rendimientos económico financieros en un mercado cada vez más competitivo. Por ello, el desarrollo de modelos de Gestión, decisión y producción, debe hacer que las CMACs sean más eficientes, y competitivas; y por lo tanto, puedan mejorar sus rendimientos. Esto, con mayor razón, si se considera el ingreso de mayores competidores, incluidos los grandes bancos, al segmento de las microfinanzas.

Objetivo General

Diseñar y formular un modelo recursivo de GHAP para optimizar los resultados económicos financieros en las CMACS del Perú.

Mediante la aplicación de un modelo recursivo de GHAP se mejorará los resultados económicos financieros.

Esto hará que las CMACS sean constructoras de valor, se puedan evaluar continuamente y garantizar su viabilidad empresarial.

\section{Hipótesis General}

Mediante un Modelo Recursivo de GHAP se logrará optimizar los resultados económicos financieros en las CMACs del Perú.

\section{MATERIALESYMÉTODOS}

La investigación es de tipo teórico- aplicada, en forma específica concierne a la Administración y Dirección de Empresas; orientado hacia la optimización de los resultados económico financieros en la CMACs del Perú.

En el presente trabajo de investigación, por su naturaleza, se utilizaron los siguientes métodos:
- DESCRIPTIVO.- El proceso del estudio y caracterización de la realidad del sistema de Cajas Municipales de ahorro y crédito en el Perú es evaluado y explicado en el presente estudio.

- $\quad$ ANAlítico - SinTÉtiCO- Los diferentes tópicos han sido analizados ampliamente mediante sus correspondientes variables los mismos que nos han permitido llegar a conclusiones valiosas al contrastar la hipótesis de trabajo; al mismo tiempo se ha llegado a conclusiones sintetizadas.

INDUCTIVO-- Mediante este método, se han inferido conceptos, teorías y conclusiones válidas para el sistema de CMACs del Perú y su desempeño dentro del sistema financiero.

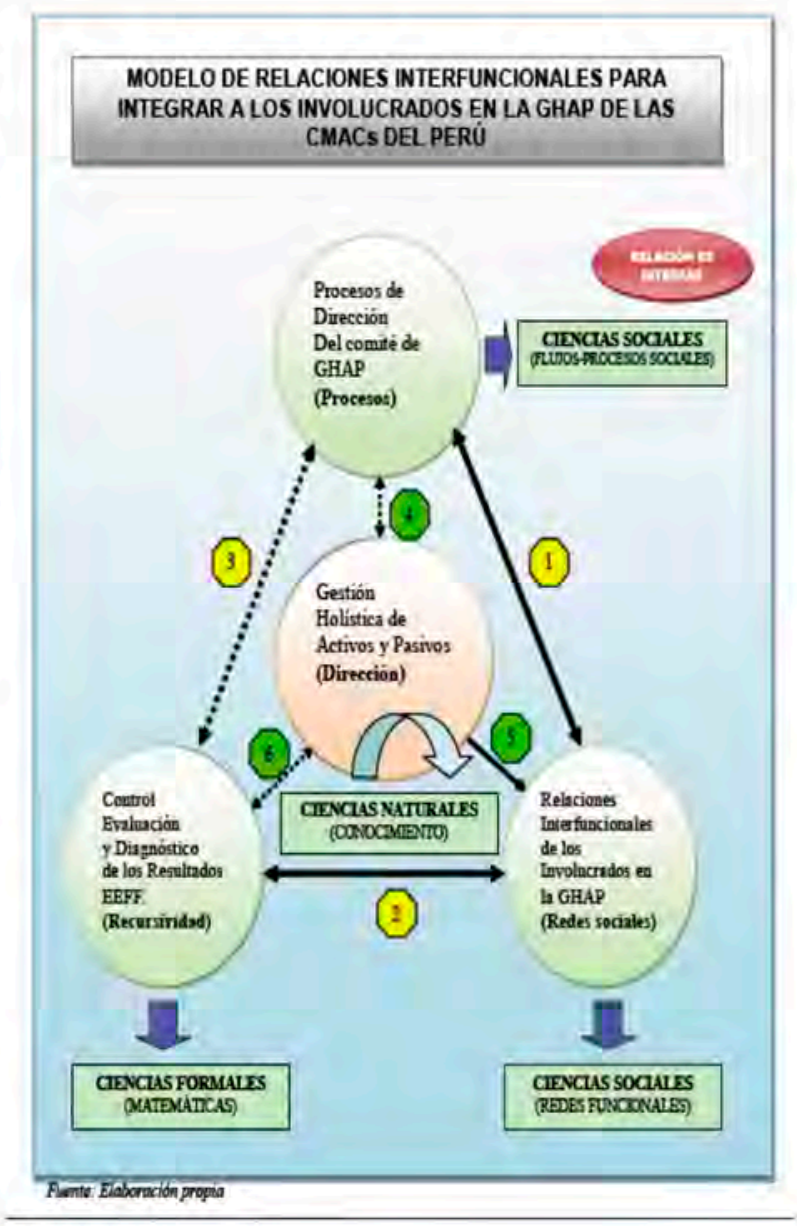




\section{RESULTADOS}

\section{PROCESAMIENTO YANÁLISIS DE DATOS}

Para la obtención de los criterios de expertos se aplicó el método Delphi a 10 expertos relacionados e involucrados en las CMACs, con nivel gerencial y con más de 05 años de experiencia. Se tomó la muestra mediante un instrumento que contiene un cuestionario orientado a verificar la necesidad de tener un modelo de Gestión, a las etapas, a los procedimientos, a los núcleos de gestión, a la participación de los involucrados y a la verificación del establecimiento de núcleos de gestión o GAP debidamente protocolizados y sistematizados.

\section{ANÁLISIS E INTERPRETACIÓN DE RESULTADOS}

Los resultados fueron los siguientes:

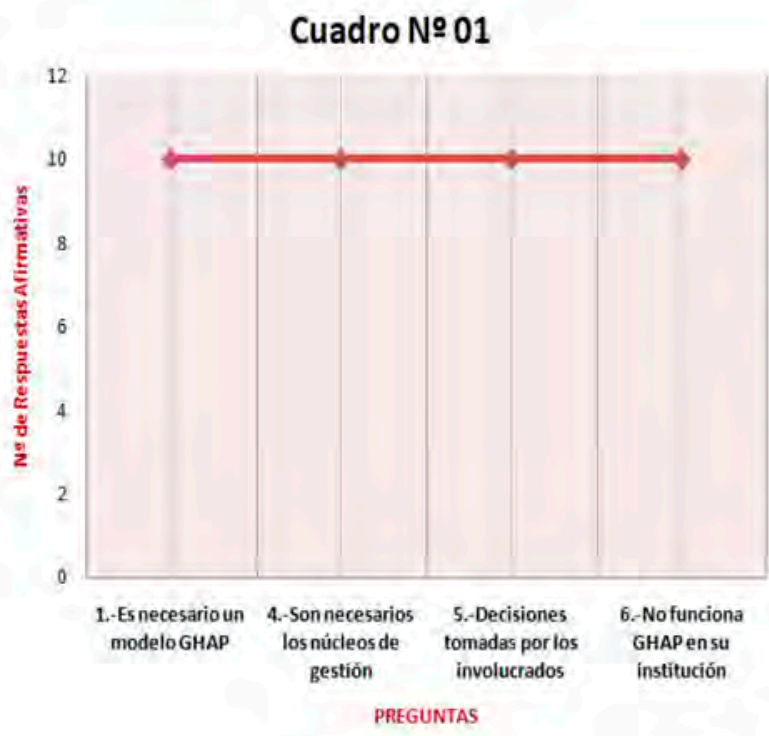

RESULTADOS DEL INSTRUMENTO No 01 PREGUNTAS CERRADAS

De los resultados podemos concluir que el MODELO GHAP propuesto en el presente trabajo de investigación, es aplicable, razonable, coherente, medible, bien estructurado, protocolizado, sistematizado, considera a los involucrados y los expertos recomiendan su aplicación. En los porcentajes de concordancia mostrados, de ellos el 100\% considera que el modelo es aplicable, razonable, coherente, que está bien estructurado y recomiendan su aplicación.

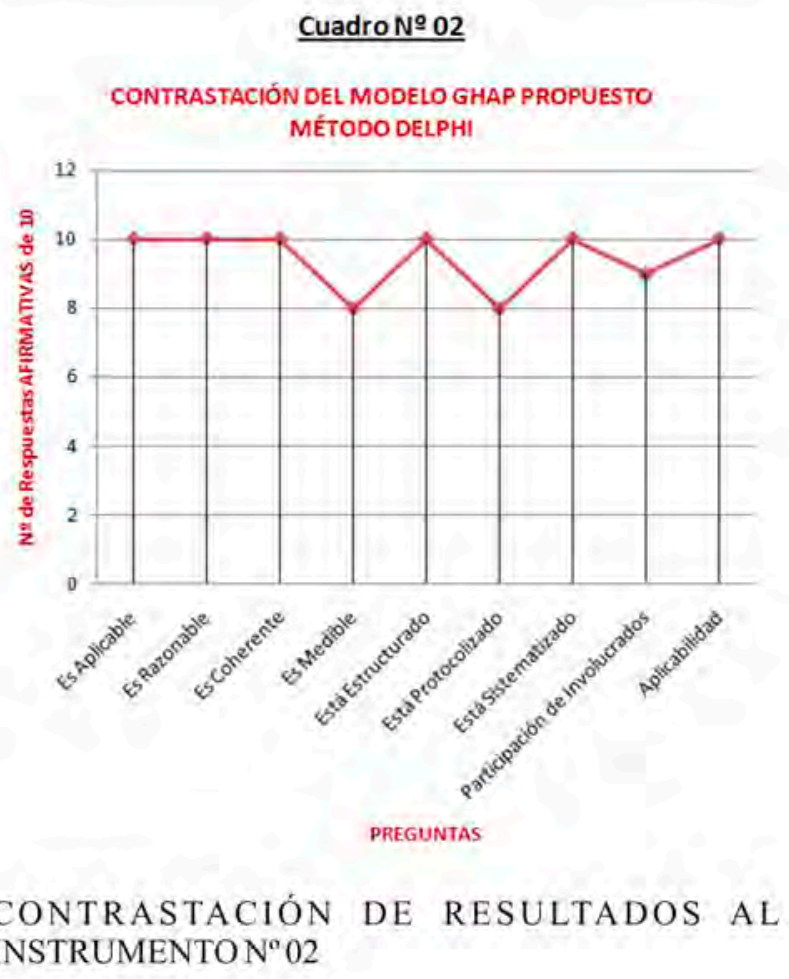

\section{REFERENCIAS}

1. Ansoff, Igor H. y Mc Donell, Edgard J. (1998) "La dirección estratégica en la práctica empresarial "Addison Wesley Longman de México S.A. de cy .Primera reimpresión en México, Traducido por Miguel Ángel Sánchez Calderón. 548 págs.

2. Apaza Meza, Mario (2005) "Contabilidad Estratégica delEVA" Editora y distribuidora Real, Perú.

3. Asobancaria, 1995, Banca y finanzas.

4. Chu Rubio, Manuel. (2007) "Fundamentos de Finanzas, un Enfoque Peruano", Lima. Impreso en los talleres de ETIPLUS SRL, $5^{a}$. Edición, 628 págs.

5. Chiavenato, Adalberto (1995)"Introducción a la teoria general de la Administración", Traducción de Germán Villamizal, Edit. Mac Graw - Hill Interamericana S.A. Cuarta Edición, Bogotá Colombia. 879 págs.

6. David, Fred R (2006) "Conceptos de Administración estratégica" México, Perrazos Educación de México Novena Edición. 336 págs. 
7. Douglas R, Emery y Jhon D, Finnerty. (2000) "Administración Financiera Corporativa", México, Editorial Pearson Educación, $1^{\text {a }}$ Edición, traducción Adolfo Deras et.al., 2000, 999 págs.

8. Eugene, Brigman Y Pappas, James. (1993) "Economía y Administración", México, Edit. McGraw Hill Interamericana de México S.A., $2^{\mathrm{a}}$ Edición, traducido por Agustín Contin, 583 págs.

9. Grant, Robert M. (1998)“Dirección Estratégica", Traducción Zulema Fernandez, Madrid, Editorial Civitas, Reimpresión primera edición 534 págs.

\section{LECTURAS}

10. PROMICRO(1999). "Principios de la filosofia de las Microfinanzas". Lectura para ser utilizada en las capacitaciones de ESAN. Tecnología Crediticia. Código HF2345.

11. Talledo Coronado P. (1999). "Filosofia y valores en el sistema de Cajas" .Lectura para ser utilizada por Universidad ESAN. Tecnología Crediticia de las Microfinanzas.

\section{DOCUMENTOS ELECTRÓNICOS}

12. Banco Multisectorial de Inversiones, May 2003,"Gestión de Activos y Pasivos". Recuperado 02.12.2009. Disponible en http://www.alide.org.pe/download/Fomin/Sem\%202 ElSalvador/Doc ElSalvador/ 12 Gestion $\% 20 \mathrm{de} \% 2$ $\overline{0}$ Activos $\% 20 \mathrm{y} \% \overline{2} 0$ Pasivos_BML_pdf

13. Federación Peruana de Cajas Municipales de Ahorro y Crédito, dic2009, "Reseña Histórica". Recuperado 02,12,2009 disponible en http://www.fpemac.org.pe/?s=institucional\&p=rese na-historica

14. Gestiopolis,(2009), "EVA, herramienta para la toma de decisiones gerenciales", Recuperado el 02.12.2009, Disponible en

http://www.gestiopolis.com/recursos/documentos/fu Ildocs/ger/EVAhptdg.htm

15. Gonzales, P. Clemente, 2009, "Parábola de los Talentos", Mateo 25,14-30. Recuperado 02.12.2009. D i s p o n i b I e e $n$ http://es.catholic,net/aprendeaorar/103/117/articulo. php?id=4I51 